\title{
Improved Bridge Method for the Measurement of Core Losses in Ferromagnetic Materials at High Flux Densities
}

\author{
William P. Harris and Irvin L. Cooter
}

\begin{abstract}
Accurate core-loss measurements at high flux densities can be made by bridge methods if the power dissipated in the primary circuit at harmonic frequencies is measured and subtracted from the apparent power dissipated in the ferromagnetic material at fundamental frequency. The determination of this harmonic power term is inconvenient, and must be done with greater accuracy than that required in the final result. An amplifier having negative output resistance was devised and is used in a manner that automatically allows accurate compensation for the harmonic power dissipation.
\end{abstract}

\section{Introduction}

In two previous papers by the present authors $[1,2]^{1}$, it was shown that accurate results can be obtained by using the a-c bridge to measure core losses in ferromagnetic materials at high flux densities only by determining and applying a "harmonic power" correction. 'The method described involved the measurement of several harmonic-frequency components, $I_{h}$, of the exciting current and the computation of the correction term as the summation of all the $I_{h}^{2} R_{p}$ terms of sufficient magnitude to affect the result, where $R_{p}$ is the total resistance of the circuit carrying the exciting current. Since terms as high as the 13 th harmonic were not uncommon, the method was complicated and rather slow, and required very accurate measurements with a sharply tuned voltmeter.

The present paper describes a method of measurement employing a power source having current feedback used in such a way as to make the circuit selfcompensating with respect to "harmonic power". The result is that accurate measurements can now be made much more conveniently than by the previous method.

\section{Theory and Principles}

The Maxwell-Wien bridge, used with an Epstein test frame, is one of several circuits used for the measurement of core losses in specimens of ferromagnetic material. This circuit is depicted in figure 1 , and the equivalent-circuit diagram is shown in figure 2 . The inductance, $L_{d}$, and the resistance, $R_{d}$, are both caused by the presence of the ferromagnetic material in the test frame, and are nonlinear because of the nature of the hysteresis cycle of the material. This nonlinearity gives rise to harmonic-frequency components in current $I$, even with source $E_{s}$ supplying a distortionless sine wave voltage. It is the power dissipated at these harmonic frequencies in the resistive elements of the circuit $\left(\Sigma I_{h}^{2} R_{p}\right)$ that causes the difficulties encountered when this circuit is used to measure core losses at high flux densities.

Previously, core losses were computed from the product $I^{2} R_{d}$, where $I$ is the rms value of the current,

${ }_{1}$ Figures in brackets indicate the literature references at the end of this paper

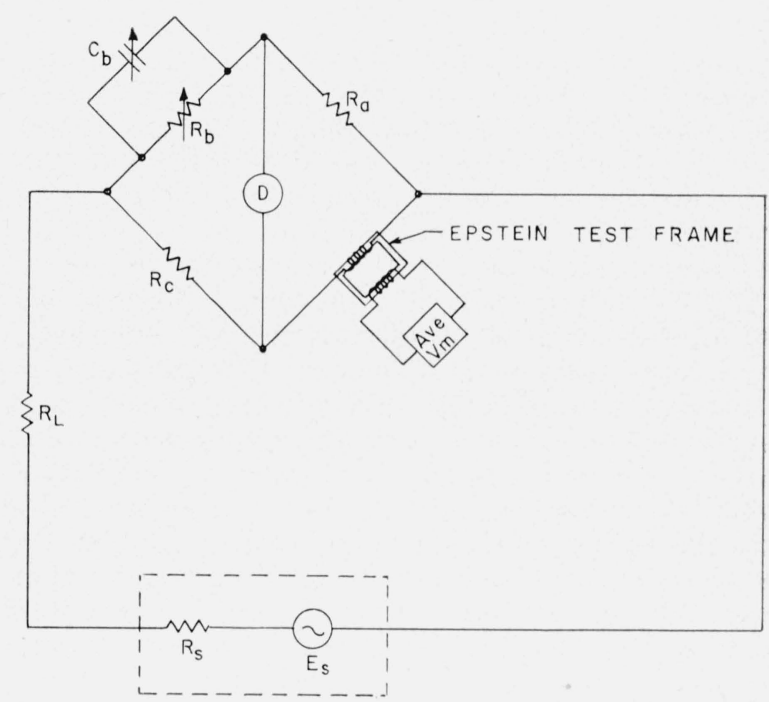

FiguRe 1. Maxwell-Wien bridge circuit for measuring core losses, using Epstein test frame.

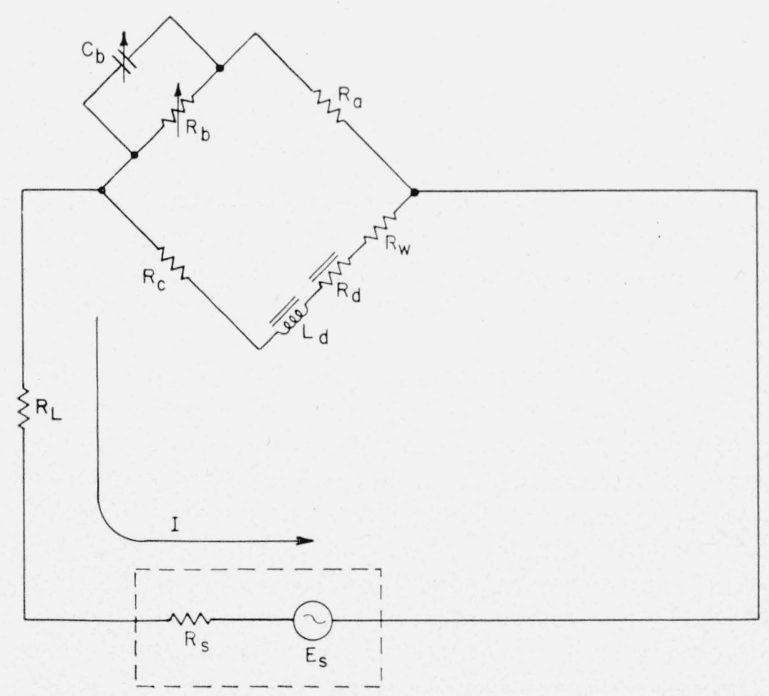

Figure 2. Equivalent-circuit diagram of the circuit shown in figure 1. 
and $R_{d}$ is the resistance associated with the ferromagnetic material in the test frame. $R_{d}$ was usually determined at fundamental frequency from the bridge balance equations and the values of $R_{a}, R_{b}$, and $R_{c}$ at balance, indicated by the tuned detector, $D$. The authors have shown $[1,2]$ that the term $I^{2} R_{d}$ includes not only the power dissipated in the iron but also all the power dissipated at harmonic frequencies in all the linear resistive elements of the circuit. The true core loss is then given by

$$
P_{c}=I_{1}^{2} R_{d}-\Sigma I_{h}^{2} R_{p}
$$

where $I_{1}$ is the fundamental frequency component of the exciting current. The correction term $\Sigma I_{h}^{2} R_{p}$ is negligible at low flux densities, but increases very rapidly at flux densities above 12 or 13 kilogausses in ordinary silicon-iron core materials, becoming several times as large as the true core losses as saturation is approached. A great burden of accuracy is thus put on the determination of the correction term. For example, if the $\Sigma I_{h}^{2} R_{p}$ is four times $P_{c}$, then to attain accuracy of 2 percent in the measurement of $P_{c}$, it is necessary to measure $\Sigma I_{h}^{2} R_{p}$ to better than 0.5 percent. The method used in the original investigation and described in the reports thereon $[1,2]$ was sufficiently accurate, but rather complicated and inconvenient. It involved the measurement of each significant harmonic current, which included harmonics as high as the 13th at the highest flux densities measured, along with the determination of $R_{p}$, taking into account the changes of resistance with changing temperature. The measurement of $R_{p}$ is further complicated by the fact that it includes the source resistance, $R_{s}$.

It was desired to devise a more convenient method of making core-loss measurements with an accuracy of 1 or 2 percent. This end would be achieved if the correction term $\Sigma I_{h}^{2} R_{p}$ could be reduced to zero. This could be done if the harmonic currents $I_{h}$, could be eliminated, or if the ohmic resistance $R_{p}$ could be reduced to zero. Although it is possible to suppress harmonics in the exciting current, this procedure is undesirable because it produces harmonics in the secondary voltage of sufficient amplitude to seriously distort the waveform. The usual practice is to strive for the conditions that will yield least distortion in the secondary voltage, and in fact measurements are ordinarily corrected to indicate what the core losses would be with a perfectly sinusoidal voltage wave.

The second alternative, the reduction of $R_{p}$ to zero, was the one used in this investigation. It is the practice to reduce the resistance of the primary circuit to a minimum in order to reduce distortion in the secondary voltage waveform. But there is a limit to how far this can be carried by the usual means, such as using heavier wire, short leads, lowervalued bridge arms, etc. In order for the bridge to be useful at all, $R_{c}$ must have a value high enough to measure conveniently. In the present work, 1 ohm was found to be the lowest practical value for $R_{c}$. The test-frame winding has appreciable resist- ance, ranging from $0.2 \mathrm{ohm}$ to several ohms for practical sizes. Leads, power source, switches, etc, unavoidably add their contributions, bringing the least practicable values of primary resistance to more than $2 \mathrm{ohms}$ in most cases. The elimination of the resistance that remains after the measures mentioned have been employed is the heart of the problem.

In order to reduce the total resistance, $R_{p}$, to zero, we have merely to add this same absolute value of negative resistance. Fortunately, this is no longer a fanciful wish. For about 25 years, electronic circuits having negative resistance properties, chiefly feedback amplifiers, have been known $[3,4,5,6,7,8,9]$. More recently the use of feedback to control the internal impedance of amplifier and other circuits has grown so widespread and so much has been published on the subject, that it is impossible to refer to all applicable publications. Those few cited should serve as a suitable point of departure for those wishing to pursue this subject.

For the present work, a high quality, 200-w amplifier was used. This amplifier had the usual feedback loops for achieving stable, distortion-free operation over a wide range of frequency. In addition, a special loop of current feedback was employed to alter the output impedance, making it possible to achieve reasonable values of negative impedance with controllable, stable operation. The essential elements of this feedback method, and the means employed to adjust it to give exactly the correct magnitude of negative resistance to counteract the positive resistance of the circuit, are described in the next section of this paper.

An alternative method of approach to the harmonic-dissipation problem is to consider the circuit as containing a series of harmonic generators, each receiving energy from the main source via the fundamental-frequency current, and delivering it to the circuit at harmonic frequency via harmonic-frequency currents. If this view is taken, the present arrangement is then a means of removing the harmonic generators from the bridge arm containing the ferromagnetic-cored test frame, putting them instead in the main power source. This view is supported by measurements of voltages at the harmonic frequencies appearing across the various elements of the circuit, with and without compensation, as described in the next section.

\section{Experimental Procedure}

\subsection{Simultaneous Measurements}

The inclusion of a wattmeter in the circuit, as described in the previous investigation $[1,2]$, provides a reference measurement made under identical conditions. This feature was found valuable, and was used in the present work. Figure 3 shows the wattmeter, $W$, with its potential coil connected to the secondary of the test frame, and its current coil in series with the bridge. It was found desirable to connect the current coil outside the bridge rather than in the arm containing the test frame, as was 


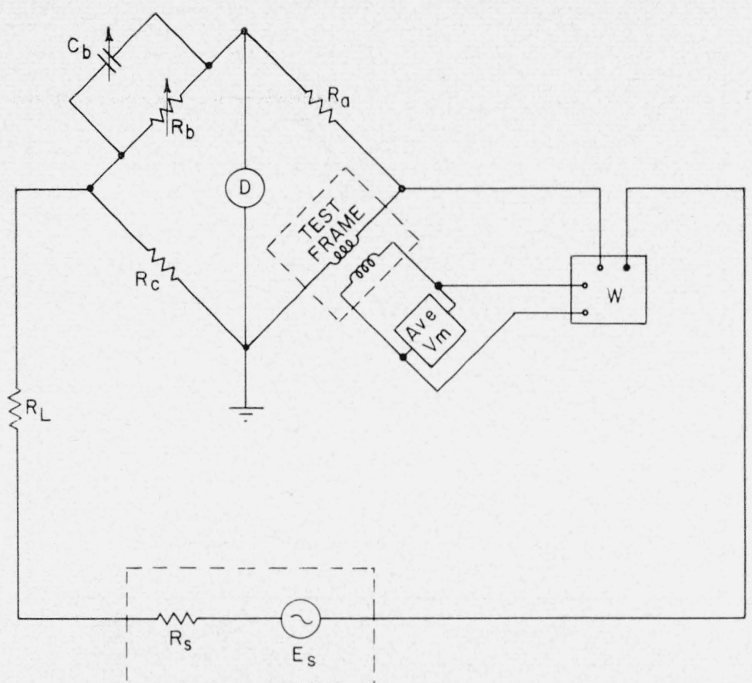

Figure 3. Method of connecting wattmeter, $W$, for making simultaneous measurements.

done in the preceding investigation. Small changes in the resistance of the current coil caused less difficulty when not included within the bridge arms. The bridge arm, $R_{a}$, was disconnected while reading the wattmeter in order to eliminate the small current in the parallel path ${ }^{2}$ through $R_{a}, R_{b}$, and $C_{b}$. All measurements were made in simultaneous pairs, and the agreement of the two methods was used as the criterion of the new method.

\subsection{Equipment}

Essential to the success of the method is the special power source used. This source must have the property of presenting an apparent internal impedance that is negative, and of precisely controllable magnitude. For precise measurements the output voltage of the source must be very stable in magnitude, because the impedance of the test frame with ferromagnetic core is a function of the current in the primary winding. Thus, at high flux densities, a small variation in source voltage causes a relatively large change in the bridge balance parameters.

To achieve the desired characteristics, a highquality power amplifier with a special feedback circuit was purchased. The basic features of the feedback circuit are shown in figure 4 . The voltage developed across a resistor in series with the primary circuit is fed back to an early stage of the amplifier, through an isolating transformer, and with means for variation by minute increments. The series resistor, $R_{1}$, can be small, 0.1 to 1 ohm, and should have low residual inductive or capacitive reactance. $R_{2}$ is a ten-turn precision potential divider, providing precise control. Variation of the position of the tap

${ }^{2}$ It was found that with $R_{a}$ not less than $30,000 \mathrm{ohms}$, the wattmeter reading was increased not more than 1 percent by connecting the current cril outside the bridge arm. Disconnecting $R_{a}$ caused the reading to be exactly the same as with the current coil in the bridge arm.

The ratio of resistances of the two current branches is seen to be at least 30,000 to 3 (at highest fiux densities), and it might be expected that the power difference would be much less than the measured 1 percent. However, the phase angles of the two currents are different, with the power factor of the large-current branch often about 0.05 , whereas that of the small-current branch is much larger. Therefore, the very small current in the latter branch contributes disproportionately to the wattmeter indication.

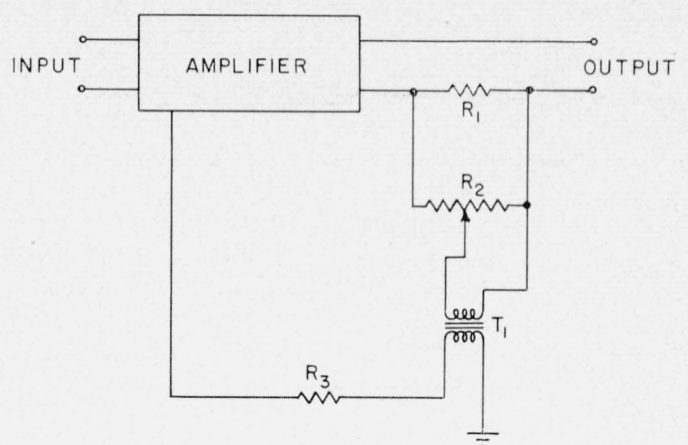

Figure 4. Basic elements of feedback loop used to control output impedance of power source.

has the effect of varying the apparent source impedance, and therefore this control will hereafter be called the impedance control, abbreviated to " $Z$ control". The transformer, $T_{1}$, is needed for isolation, and should be capable of handling a range of frequencies wide enough to include the fundamental and all significant harmonics with a minimum of phase shift or attenuation. In practice, a small amount of phase shift in the fed-back voltage may be corrected by a simple $R$ - $C$ correction network.

The signal input to the amplifier was from a lowdistortion oscillator. A frequency of 70 cps was chosen for these tests, in order to eliminate the question of pickup and interference from the powerline frequency.

The test frame was a 25-cm Epstein frame, with primary and secondary of 700 turns each. For this investigation the frame was used without an airflux-compensating mutual inductor. The values of flux density, $B$, are therefore slightly too high, but this does not affect the validity of the comparison, because both the wattmeter and bridge methods were used simultaneously.

The bridge-balancing components, $R_{b}$ and $C_{b}$, were high-quality decade boxes, with accurately known values and negligible residual reactances. $R_{a}$ was a precision resistor of carefully determined value. $R_{c}$ was a precision resistor of $250-\mathrm{w}$ rating. Lowerpowered resistors might be used, provided they would carry current up to 2 amp without changing resistance.

In order to determine the core loss, it is necessary to measure the value of the fundamental-frequency current in the test frame. This can be determined from the fundamental-frequency voltage drop across $R_{c}$. The accuracy necessary in this measurement can be readily obtained with the polar potentiometer method [10], which was used for these measurements. The method requires a reference voltage of the same frequency as the unknown, variable in phase and amplitude, with means for accurate determination of the amplitude. Figure 5 depicts the circuit used for this purpose. A signal from the source driving the main amplifier is fed to a phase shifter, capable of shifting the phase by any amount required, with fine control. The phase shifter introduces distortion, which is removed by the filter. The transformer, $T_{2}$, is shown to emphasize the need for complete 


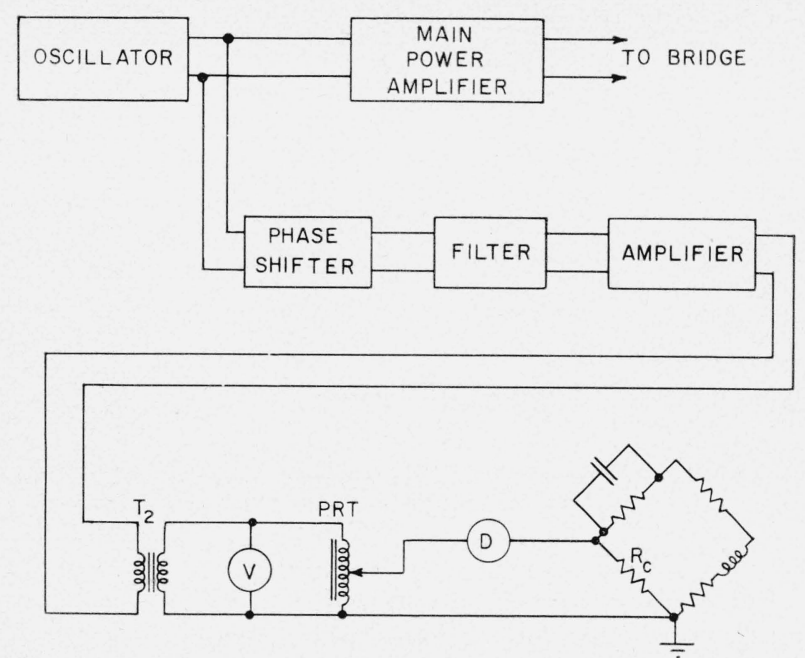

Figure 5. Polar potentiometer used to measure the voltage drop across $R_{c}$.

isolation. A feedback connection to the output side of the output transformer is often found on power amplifiers, and this could render the apparatus unusable unless isolation is employed. Isolation is especially important for measurement of the voltage drop across a resistor, if both ends of the resistor are operating at nonground potentials.

The accuracy of the method is determined largely by the next two elements, the reference voltmeter, $V$, and precision ratio transformer, PRT. The variable-phase reference voltage is applied to the primary of the $P R T$, and measured by the reference voltmeter, $V$. The PRT used has five decade switches, by which the output voltage may be varied in steps as small as $1 \times 10^{-5}$ times the input voltage. The voltmeter, $V$, was adjusted to have a zero scale correction at the reference input voltage, and care was taken to maintain the input at this level throughout the measurements. All measured voltages were then determined in terms of a ratio, read from the $P R T$, times the reference voltage. The accuracy of the measurement of the fundamental-frequency current is estimated to be within \pm 0.2 percent.

In use, the phase shifter and the PRT are adjusted so that the detector, $D$, tuned to the fundamental frequency, reads a null. Under these conditions, the output from the PRT matches the fundamental component of the unknown in phase and amplitude.

It is obvious that the apparent loss measured by the bridge is profoundly affected by the setting of the $Z$ control. One of the major problems of this investigation was to devise a means of setting the $Z$ control at the proper point.

It is relatively easy to find the proper setting to compensate for those elements of the circuit whose terminals are accessible. This is evident from a consideration of figure 6 , which shows the basic elements of such a circuit as that with which we are concerned. If a tuned detector, such as a wave analyzer, is connected to points 1 and 2 , and tuned to the third harmonic of the fundamental frequency,

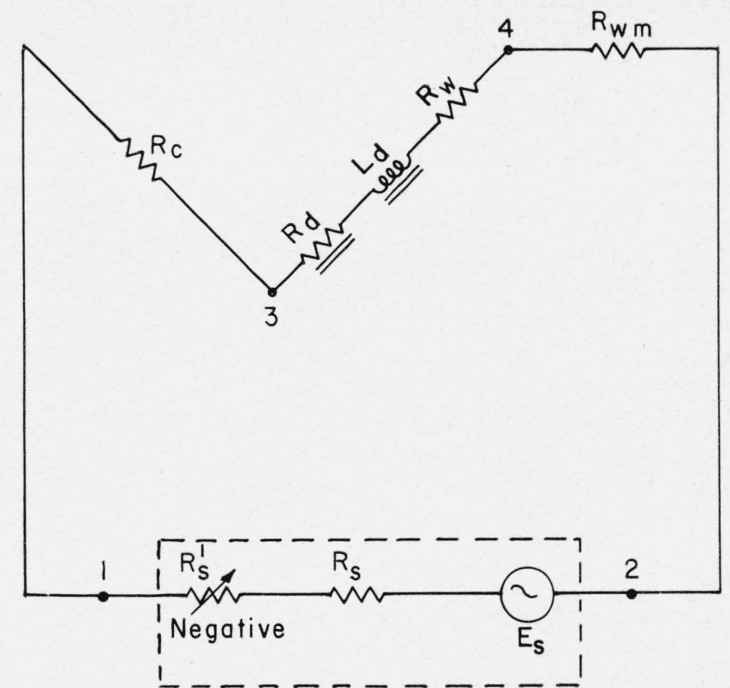

FIGURE 6. The primary circuit, showing how compensation for part of the circuit resistance may be achieved by varying the negative source resistance, $R_{s}^{\prime}$.

a setting of the $Z$ control can be found that will yield a minimum deflection of the detector. This is done with the amplifier supplying a current large enough to produce considerable harmonic components. At this setting, the source resistance, $R_{s}$, has been compensated by making $R_{s}{ }^{\prime}$ equal in magnitude and opposite in sign to $R_{s}$. If now the detector is moved to points 2 and 3 , and again the $Z$ control is adjusted to produce a minimum deflection, the resistor, $R_{c}$, is also compensated, and $R_{s}{ }^{\prime}=-$ $\left(R_{s}+R_{c}\right)$. By connecting to points 3 and 4 , we can compensate for $R_{s}, R_{c}$, and $R_{w m}$. But here this method ends, for the resistance of the primary winding of the test frame, $R_{w}$, which remains to be compensated, is not separately available to us; it is associated with $L_{d}$ and $R_{d}$ between the terminals of the test frame.

This problem was solved by making a resistor, $R_{w}{ }^{\prime \prime}$, exactly equal in resistance ${ }^{3}$ to $R_{w}$, and insert-

${ }^{3} R_{w}$ is the ohmic or d-c resistance of the test frame primary winding. Therefore, $R_{w}$ " is a "pure" resistance, i. e., with negligible inductive or capacitive reactance. The d-c and a-c resistance of $R_{w}$ and $R_{w}$ " are equal.

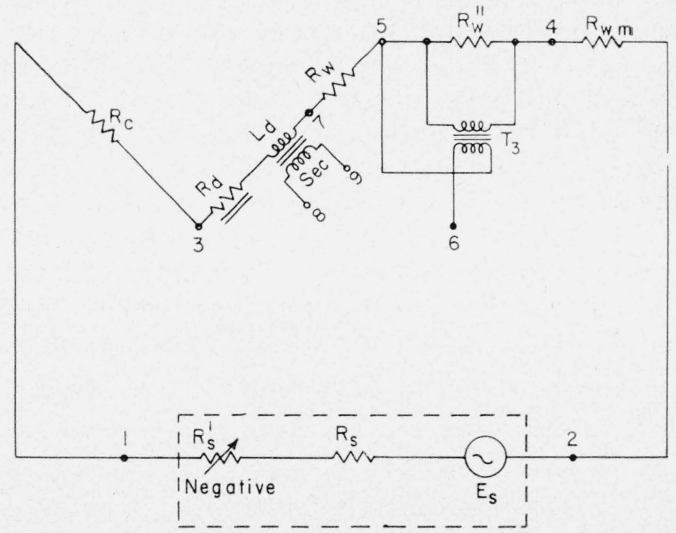

Figure 7. Circuit with $R_{w}{ }^{\prime \prime}$ added, by means of which complete compensation may be achieved. 
ing it in the circuit as shown in figure 7 . If the detector were connected to points 3 and 5 , and the $Z$ control adjusted for minimum deflection, compensation would be effected for $R_{s}, R_{c}, R_{w m}$, and $R_{w}{ }^{\prime \prime}$. If we could connect the detector to point 3 and the phantom point 7 , we could achieve complete compensation for all the ohmic resistance of the circuit.

The transformer, $T_{3}$, provides the means for a virtual connection to the phantom point 7 . The ratio of this transformer is precisely unity, and its phase shift negligible. The secondary can be connected in such a way that the voltage induced in it opposes the $I R$ drop in $R_{w}$. Since the voltage on the primary of $T_{3}$ is the $I R$ drop in $R_{w}{ }^{\prime \prime}$, which was made to equal $R_{w}$, the induced voltage just equals the $I R$ drop in $R_{w}$, and by connecting to 3 and 6 , we get the same effect as if we were able to connect to 3 and 7 . All that remains is to adjust the $Z$ control for a minimum deflection.

The method of setting the $Z$ control, using the third harmonic produced by the iron, leaves something to be desired. The main power amplifier inevitably produces some harmonic distortion in its own circuit, and this is indistinguishable from that produced by the iron, so that the final setting of the $Z$ control is affected by an unwanted factor. The method yields usable results if the amplifier distortion is kept low enough, but this is a difficult task at the power levels required for testing.

An alternative method of setting the $Z$ control was devised, which avoids the difficulty pointed out above. All that is required is a voltage, to be introduced into the circuit at some point external to the main amplifier without changing the ohmic resistance of the circuit. This can be achieved in practice by applying a voltage to the secondary winding of the test frame, with the specimen in the frame acting as the core of a transformer, and inducing a voltage in the primary winding of the frame. The detector, connected as before, is tuned to the frequency of the injected voltage, and the $Z$ control set by this means.

It was found that the setting of the $Z$ control was slightly dependent upon the amplitude of the injected voltage. Best results were obtained by adjusting this amplitude to yield about the same rms current in the primary circuit with only the injected voltage, operating through the feedback circuit (i. e., no input to the main amplifier input terminals) as the rms current existing under testing conditions. That is, for a test point requiring exciting current of 1 amp rms, the $Z$ control was set with the injected voltage adjusted to give 1 amp rms in the primary circuit. The difference in $Z$ control settings made with various injected voltages was small, however, and could be neglected if errors approaching 3 to 5 percent were allowed.

An obvious advantage of this latter method is that the frequency of the injected voltage may be chosen at will. By choosing several frequencies covering the range of the predominant harmonics of the frequency used for the tests, it was determined that the setting of the $Z$ control was practically the same for all frequencies in this range (50 to $700 \mathrm{cps}$ ). If this were not so, it would indicate that the equipment was not suitable for use in this manner, for the predominant harmonics must all be compensated simultaneously in order to get accurate results. In practice, a frequency of 200 to 350 cps was used.

It is necessary to consider the effect on the primary circuit resistance of the transferred impedance of the auxiliary circuit supplying the injected voltage. If this transferred impedance altered the net resistance of the primary circuit, then the $Z$ control would be set wrongly. But no errors arise here, because as the $Z$ control approaches the correct setting, the net resistance of the primary circuit approaches zero, and the resistive component of the transferred impedance paralleling this zero resistance does not alter the total. This was verified experimentally. Various taps of the transformer in the auxiliary injection circuit were tried, and various loads connected across the frame between points 8 and 9 of figure 7 . In all cases, the setting of the $Z$ control was unaffected. That this setting also yielded correct core-loss measurements is further proof that no significant error arose from this source.

When the distortion introduced by the main power amplifier was less than about 0.1 percent, the injected-voltage method of setting the $Z$ control agreed with the method using the iron-produced harmonics as described previously. Both methods were used to obtain the data reported herein.

To obtain the power loss in the iron, the value of $R_{d}$, which is the apparent increase in the resistance of the frame, is required. Unavoidably, however, the ohmic resistance of the primary winding of the test frame, $R_{w}$, is included in the measured arm of the bridge. Two methods are available to separate these two components of resistance. $R_{w}$ can be determined independently and subtracted from the value obtained from the bridge measurement. Alternatively, the ohmic resistance can be compensated by an auxiliary component in parallel with the balancing arm of the bridge, as devised by Dieterly and $W$ ard [11]. The two methods give identical results, and both were used successfully during the course of this investigation.

The complete circuit is shown in figure 8 . Selector switches are shown in proper position for balancing the bridge.

\subsection{Specimens}

Each specimen weighed approximately $500 \mathrm{~g}$ and consisted of strips $3 \mathrm{~cm}$ wide and $28 \mathrm{~cm}$ or $30.5 \mathrm{~cm}$ long. Five grades of nonoriented silicon sheets and one grade of oriented-grain material were used for these tests.

\subsection{Measurements}

With harmonic power losses fully compensated by means of the feedback circuit, the power loss in the iron is simply

$$
P_{d}=I_{1}^{2} R_{d} \text {. }
$$

Thus, there are but two quantities to measure, $I_{2}$ and $R_{d}$. 


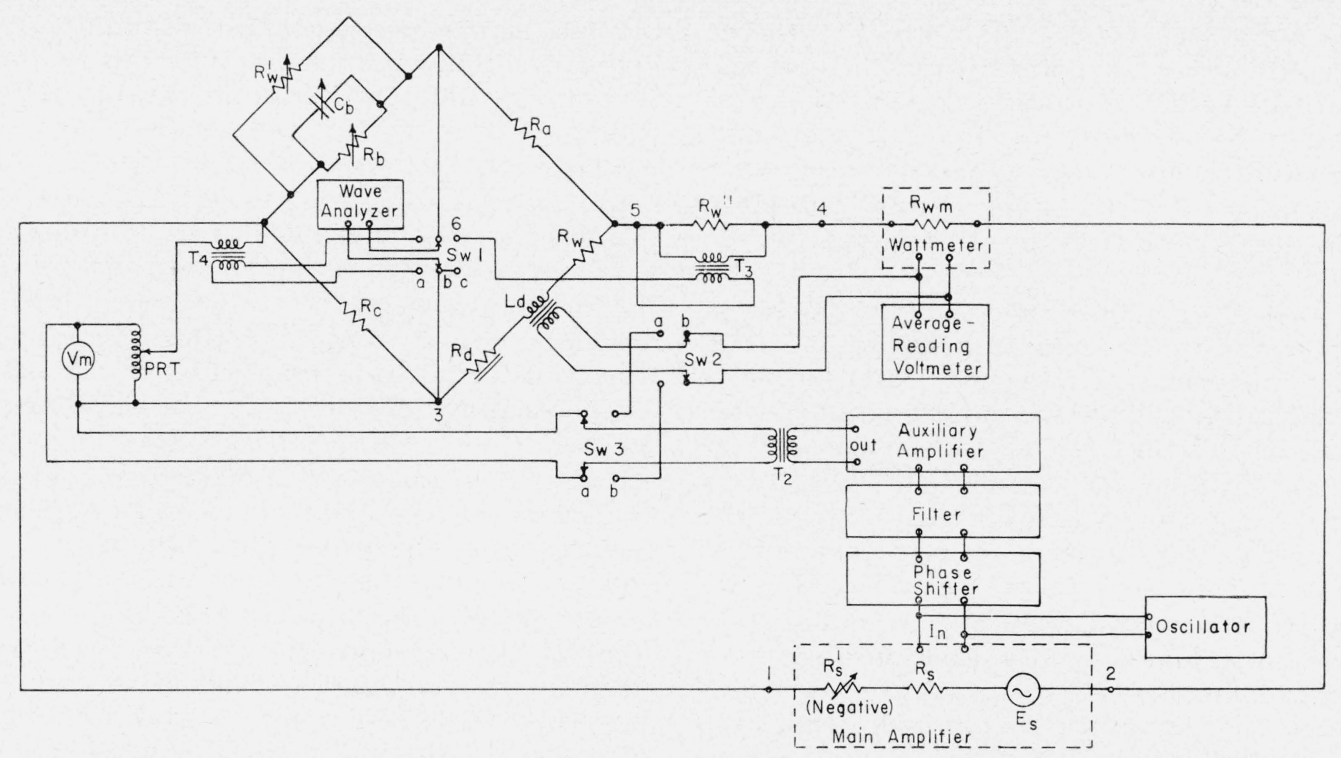

FIGURE 8. Complete circuit for making core-loss measurements with self-compensating bridge method.

Before these measurements can be made, however, the $Z$ control must be set accurately. Therefore, the procedure described in the next paragraph was followed.

The resistor, $R_{w}^{\prime \prime}$, (see fig. 8) was adjusted ${ }^{4}$ to match $R_{w}$. $R_{w}^{\prime}$, if used, was set to compensate ${ }^{5}$ for $R_{w}$. The specimen was then inserted in the frame, and a signal applied to the secondary winding of the frame ( $\mathrm{Sw}_{w} 2$ in position a, $\mathrm{S}_{w} 3$ in position b), with no input to the main amplifier. The detector, tuned to the injected-signal frequency, was connected to points 3 and 6 of figure 8 ( $\mathrm{Sw} 1$ in position c). The $Z$ control was adjusted to yield a minimum deflection of the detector. The detector was then switched to the main bridge ( $\mathrm{SW}_{\mathrm{w}} 1$ in position $\mathrm{b}$ ), and the secondary of the test frame connected to the wattmeter and voltmeters ( $\mathrm{Sw} 2$ in position b). A voltage was applied through the main amplifier, and the main bridge balanced by means of $R_{b}$ and $C_{b}$. The polar potentiometer was then used ( $\mathrm{S}_{\mathrm{w}} 3$ in position a, $\mathrm{S}_{w} 1$ in position a) to measure the voltage at fundamental frequency appearing across $R_{c}$. The rms voltmeter connected to the secondary of the test frame was read, in order to be able to determine the form factor of the voltage waveform. A reading from the average-indicating voltmeter, used to determine the form factor and flux density, and a reading from the reference wattmeter (with $R_{a}$ disconnected) completed the measurements.

${ }^{4}$ It was found that the highest current used in this investigation, $2 \mathrm{amp}$ caused the windings to rise $10^{\circ} \mathrm{C}$, causing a change in resistance too large to neglect. Therefore, a chart was prepared to indicate the setting of a fine control on $R_{u}^{\prime \prime}$, corresponding to the temperature of $R_{v}$, read from a thermometer held in close contact to $R_{w}$. A better method might be to incorporate another winding on the test frame to be used as a resistance thermometer in a very-lowcurrent d-c bridge circuit.

A similar chart was prepared to determine the setting of $R_{w}^{\prime}$ as a function of temperature.

${ }_{5}$ Dieterly and Ward [11] showed that if $R_{w}^{\prime}$ (fig. 8) is made equal to $R_{a} R_{c} / R_{w}$ then $R_{d}=R_{a} R_{d} / R_{b}$. This procedure obviates the necessity of subtracting $R$ from each resistance measurement made, for if $R_{w}^{\prime}$ is not nsed, then $R_{d}=$ $\left(R_{a} R_{c} / R_{b}\right)-R_{w}$

\section{Results}

The results of a series of measurements are given in table 1. Each determination of core loss by the compensated-bridge method is accompanied by the reference value from the wattmeter method. The last column gives the difference between the two methods. It will be noted that the greatest difference is 2.5 percent, and that only 6 of the 46 pairs differ by as much as 1.0 percent.

These same results are given in graphic form in figure 9 , where it is evident that excellent agreement is obtained.

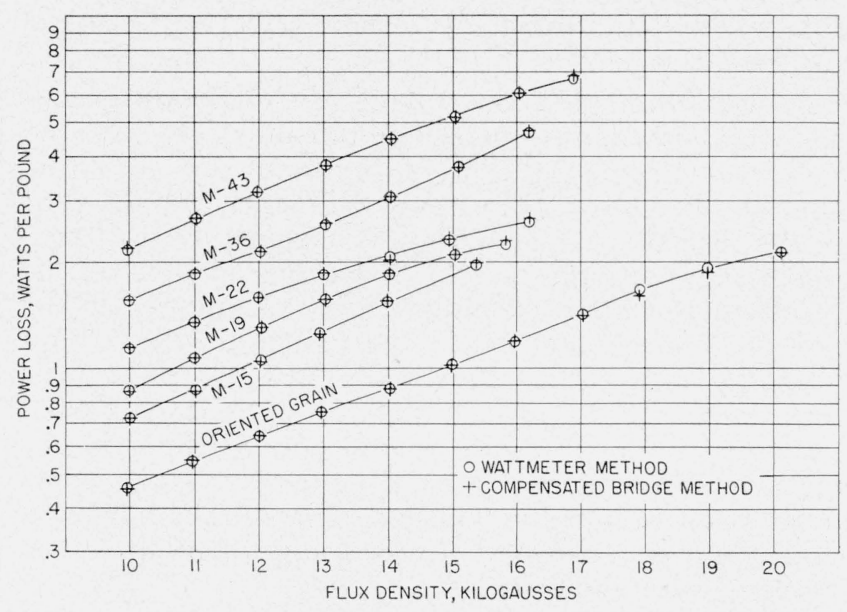

FIGURE 9. Comparison of power-loss measurements made with compensated bridge and wattmeter, at $70 \mathrm{cps}$.

No corrections were made for instrument loss or form factor errors, which are identical for the two methods. 
Figure 10 shows the magnitude of the contribution of the compensation method. For comparison, a series of measurements was made with the circuit unchanged, except that no feedback for compensation was used. The uncorrected, or apparent, core loss, $I_{1}^{2} R_{d}$, is shown as the upper curve, diverging more and more (as flux density is increased) from the true core loss, shown as the lower curve. As pointed out in a previous paper [2], the uncorrected bridge gives results that may be as much as several hundred percent too high.

The power-loss data are not corrected for losses in the measuring instruments (the wattmeter potential coil and the flux voltmeter), or for the errors caused by the departure of the flux waveform from a true sinusoid. These corrections are identical for the two methods, and thus do not affect the validity of the comparison.

TABLE 1. Comparison of power loss measured by compensated bridge and wattmeter, at $70 \mathrm{cps}$

INo corrections were made for instrument loss or form factor errors. These are identical for the two methods, and do not affect the validity of the comparison]

\begin{tabular}{|c|c|c|c|c|c|c|}
\hline \multirow{2}{*}{ Specimen } & \multirow{2}{*}{ Gage } & \multirow{2}{*}{$\begin{array}{c}\text { Effective } \\
\text { weight }\end{array}$} & \multirow{2}{*}{$B$} & \multicolumn{2}{|c|}{ Power loss } & \multirow{2}{*}{$\Delta$} \\
\hline & & & & Bridge & $\begin{array}{l}\text { Watt- } \\
\text { meter }\end{array}$ & \\
\hline $\begin{array}{l}\text { Oriented } \\
\text { grain. }\end{array}$ & 29 & 0.849 & $\begin{array}{c}\begin{array}{c}\text { Kilo- } \\
\text { gausses } \\
9.98 \\
10.98 \\
12.00 \\
12.99 \\
14.01 \\
15.00 \\
15.98 \\
17.02 \\
17.96 \\
18.99 \\
20.12\end{array}\end{array}$ & $\begin{array}{r}w / l b \\
0.449 \\
.540 \\
.642 \\
.749 \\
.875 \\
1.018 \\
1.203 \\
1.425 \\
1.643 \\
1.888 \\
2.128\end{array}$ & $\begin{array}{r}w / l b \\
0.452 \\
.538 \\
.641 \\
.749 \\
.874 \\
1.020 \\
1.19 i \\
1.435 \\
1.685 \\
1.925 \\
2.140\end{array}$ & $\begin{array}{r}\% \\
-0.7 \\
+.4 \\
+.2 \\
.0 \\
+.1 \\
-.2 \\
+.5 \\
-.7 \\
-2.5 \\
-1.9 \\
-0.6\end{array}$ \\
\hline $\begin{array}{l}\text { Nonoriented: } \\
\qquad \text { M-15 }\end{array}$ & 29 & 0.903 & $\begin{array}{l}10.01 \\
11.00 \\
12.02 \\
12.95 \\
13.99 \\
15.38\end{array}$ & $\begin{array}{r}0.714 \\
.861 \\
1.042 \\
1.258 \\
1.543 \\
1.988\end{array}$ & $\begin{array}{r}0.715 \\
.868 \\
1.049 \\
1.271 \\
1.539 \\
1.973\end{array}$ & $\begin{array}{r}-0.1 \\
-.8 \\
-.8 \\
-1.0 \\
+0.3 \\
+.8\end{array}$ \\
\hline M-19. & 29 & 0.744 & $\begin{array}{l}10.00 \\
11.00 \\
12.02 \\
13.00 \\
14.01 \\
15.02 \\
15.83\end{array}$ & $\begin{array}{l}0.866 \\
1.060 \\
1.293 \\
1.553 \\
1.835 \\
2.094 \\
2.299\end{array}$ & $\begin{array}{l}0.869 \\
1.060 \\
1.294 \\
1.560 \\
1.848 \\
2.096 \\
2.257\end{array}$ & $\begin{array}{r}-0.3 \\
.0 \\
-.1 \\
-.4 \\
-.7 \\
-.1 \\
+1.9\end{array}$ \\
\hline M-22 & 26 & 0.836 & $\begin{array}{l}10.00 \\
11.00 \\
12.00 \\
13.00 \\
14.03 \\
14.95 \\
16.21\end{array}$ & $\begin{array}{l}1.127 \\
1.337 \\
1.578 \\
1.856 \\
2.024 \\
2.332 \\
2.653\end{array}$ & $\begin{array}{l}1.124 \\
1.340 \\
1.581 \\
1.849 \\
2.067 \\
2.330 \\
2.622\end{array}$ & $\begin{array}{r}+0.3 \\
-.2 \\
-.2 \\
+.4 \\
-2.1 \\
+0.1 \\
+1.2\end{array}$ \\
\hline M-36 & 26 & 0.877 & $\begin{array}{l}10.00 \\
11.01 \\
12.01 \\
13.02 \\
14.03 \\
15.10 \\
16.19\end{array}$ & $\begin{array}{l}1.544 \\
1.854 \\
2.147 \\
2.572 \\
3.051 \\
3.731 \\
4.720\end{array}$ & $\begin{array}{l}1.544 \\
1.847 \\
2.148 \\
2.558 \\
3.055 \\
3.75 \\
4.70\end{array}$ & $\begin{array}{r}0.0 \\
+.4 \\
.0 \\
+.5 \\
-.1 \\
-.5 \\
+.4\end{array}$ \\
\hline M-43 & 24 & 0.895 & $\begin{array}{r}9.98 \\
11.02 \\
11.99 \\
13.03 \\
14.04 \\
15.03 \\
16.04 \\
16.88\end{array}$ & $\begin{array}{l}2.179 \\
2.674 \\
3.183 \\
3.774 \\
4.442 \\
5.194 \\
6.049 \\
6.747\end{array}$ & $\begin{array}{l}2.171 \\
2.658 \\
3.181 \\
3.775 \\
4.455 \\
5.194 \\
6.065 \\
6.69\end{array}$ & $\begin{array}{r}+0.4 \\
+.6 \\
+.1 \\
-.0 \\
-.3 \\
-.0 \\
-.3 \\
+.9\end{array}$ \\
\hline
\end{tabular}

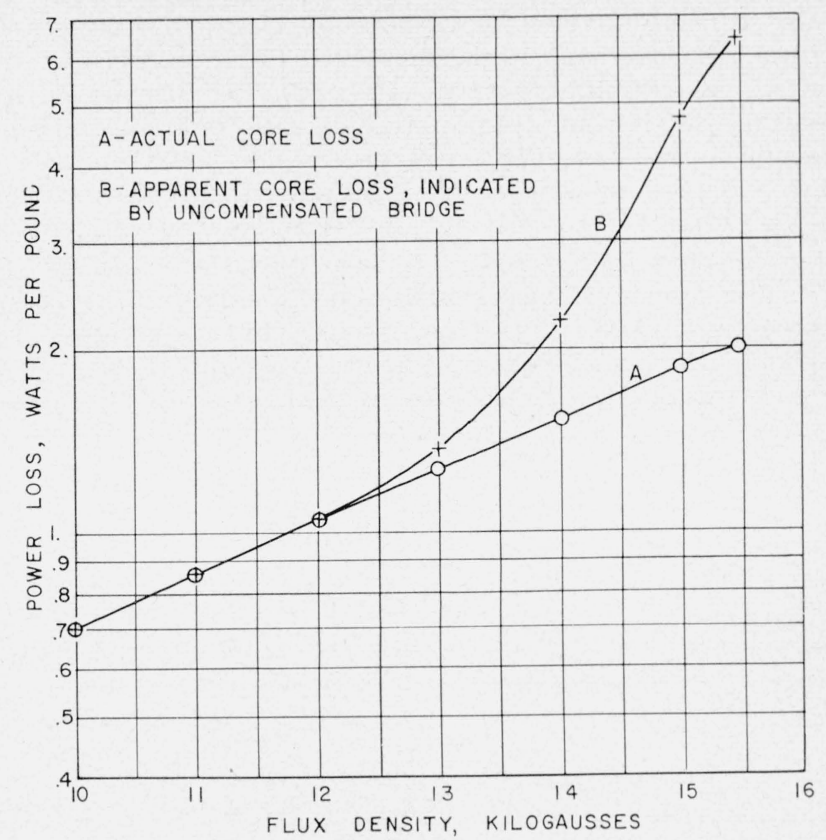

Figure 10. Apparent power loss as measured by uncompensated bridge compared to actual core loss, at 70 cps.

\section{Summary and Conclusions}

In order to obtain accurate measurements of core loss by the bridge method, the harmonic power dissipated in the resistive elements of the entire circuit must be taken into account. If each harmonic component must be measured and computed separately, the procedure becomes inconvenient and time consuming. A compensation method, using an electronic power amplifier with "current" feedback, has been devised and found to be sufficiently accurate.

The principal difficulty encountered was the finding of a method of adjusting the feedback to just the correct amount to effect complete compensation, but not under- or overcompensation. 'This problem was solved, and the method was successfully developed to yield results that differ from those obtained from the wattmeter by not more than 2.5 percent in the worst cases, with most measurements agreeing to 1 percent or better.

It is estimated that the determination of the fundamental-frequency current is accurate to \pm 0.2 percent. The variation of the output voltage of the power source was the limiting factor in the precision of balance of the bridge. The nonlinearity of the test frame with ferromagnetic core causes the balance parameters to be strongly dependent on the impressed voltage. At highest flux densities, the precision of balance is estimated to be about 0.5 percent for the equipment used.

The accuracy limitations are seen to be associated with the equipment employed and not inherent in the method. Therefore, further refinement of the power source, including the current feedback circuit for the $Z$ control, and of the method of measuring 
the fundamental-frequency current would result in more precise and more accurate measurements.

It is concluded that the use of feedback as described in this paper makes the bridge method capable of producing accurate results with a reasonable degree of convenience, even at high flux densities, where distortion in the exciting current would cause very large errors if not taken into account.

The method was developed by using materials, specimen sizes, and frequencies that were easy to check with the wattmeter method, but its greatest usefulness will probably be in those ranges where the wattmeter is unsuitable.

Washington, November 29, 1957.

\section{References}

[1] I. L. Cooter and W. P. Harris, The use of an a-c bridge to measure core loss at high inductions, Trans. Am. Inst. Elec. Engrs. 75, Part 1, 252 (1956).

[2] I. L. Cooter and W. P. Harris, Investigation of an alternating-current bridge for the measurement of core losses in ferromagnetic materials at high flux densities, J. Research NBS 57, 103 (1956) RP2699.

[3] H. F. Mayer, Control of the effective internal impedance of amplifiers by means of feedback, Proc. (Inst. Radio Engrs.) 27, 213 (1939).

[4] H. Bartels, Graphische Berechnung linearer und nichtlinearer Rückkopplungen, Elek. Nach. Tech. 11, 319 (1934).

[5] T. Sturm, Verfahren zur Klirrverminderung (Linearisierung), Funktechnische Monatshefte, p. 77 (1937).

[6] E. L. Ginzton, Balanced feed-back amplifiers, Proc. (Inst. Radio Engrs.) 26, 1367 (1938).

[7] E. L. Ginzton, Stabilized negative impedances. Electronics 18. In three parts: Part 1, p. 140 (1945); Part 2, p. 138 (1945); Part 3, p. 140 (1945).

[8] H. W. Bode, Network analysis and feedback amplifier design (D. Van Nostrand, New York, N. Y., 1945).

[9] J. G. Thomason, Linear feedback analysis (MícGrawHill Publishing Co., Inc., New York, N. Y., 1955).

[10] F. K. Harris, Electrical measurements (Wiley, New York, N. Y., 1952)

[11] D. C. Dieterly and C. E. Ward, A wide-range a-c bridge test for magnetic materials, Am. Soc. Testing Materials Bul. 182 (1952). 